\title{
FOUCAULT Y RANCIÈRE: SUBJETIVIDAD Y POLÍTICA
}

\author{
Carlos Ossandón B. \\ Universidad de Chile \\ cob2002@u.uchile.cl
}

\begin{abstract}
ReSUMEN / ABSTRACT
En el marco de las complicidades que en distintos ámbitos se pueden identificar entre las obras de Foucault y Rancière, el presente análisis revisa más particularmente el modo cómo ambos autores abordan los vínculos entre subjetividad y política.
\end{abstract}

Palabras Clave: Foucault, Rancière, subjetividad, política.

\section{FOUCAULT AND RANCIÈRE: SUBJECTIVITY AND POLITICS}

Abstract: In the framework of the complicities that can be identified in different fields between Foucault's and Rancière's works, the present analysis reviews more particularly the way in which both authors approach the links between subjectivity and politics.

KEYWORDS: Foucault, Ranciére, Subjectivity, Politics.

$\mathbb{R A}$

I. Se ha señalado que entre Foucault y Rancière habría una "complicidad en el pensar" . No es el caso detenerse demasiado en aspectos que son muy conocidos y que sustentan prácticamente esta "complicidad": me refiero a la "atmósfera" social y política de las décadas de 1960 y 1970 y, en particular, a mayo 68, circunstancia esta última -se ha dicho-que va a modificar objetos, introducir temas poco apreciados por el pensamiento político e incluso replantear proyectos de vida. Se da en este periodo un espectro de tópicas que formarían parte de un particular "momento" o "aventura" de la filosofía francesa del siglo XX (Badiou 2010 y 2014). En Foucault y Rancière, este "momento" expresa el afán común de emprender nuevos derroteros críticos, no desligados de la investigación y de la acción o de la conjunción de ambas, y cuyo interés por el presente o por un tipo de exploración inmanente, que ya no recurre ni descansa en filosofías de la historia o en fundamentos últimos, es más fuerte o reemplaza la adscripción sin más a una escuela.

Pero hay más. Alain Badiou señala que hay al menos dos rasgos característicos de la filosofía que se practica en Francia. En primer lugar, la dimensión política, cuestión que se expresa en un pensamiento que desde sus propios recursos, y bajo un 
horizonte antiautoritario y antidisciplinario, busca acompañar las transformaciones de la subjetividad $^{2}$. En segundo lugar, la relevancia que adquiere la pregunta por la escritura que conviene a la filosofía, en concomitancia esta pregunta con la relación que establece con distintas "formas" de las artes. Este vínculo con las artes y la literatura, sin ser el único nexo a destacar, sería -según Badiou- una de las características más notables de la filosofía francesa en el siglo XX $(2014$, p. 15); característica que va a redundar en "la cuestión de la forma de la filosofía misma" (Badiou 2010, p. 84). El interés por la literatura es algo más que un objeto exterior a la filosofía: ella a la vez que precipita "afueras" o transgresiones se cuela desde dentro determinando o haciendo del estilo o de la propia escritura filosófica una posibilidad de pensamiento para la filosofía misma. Así las obras literarias no solo movilizan o vehiculizan desde sus propias configuraciones o juegos lingüísticos el pensamiento (Badiou 1998 y 2007) sino también desafían en este mismo plano configurador o formal a la filosofía misma. Termina diciendo Badiou: "Casi se podría decir que una de las metas de la filosofía francesa era crear un nuevo lugar de escritura, en el cual la literatura y la filosofía fueran indiscernibles" (2014, p. 16). Foucault y Rancière no son ciertamente ajenos, con resultados distintos, a esta tradición y aspiración señalada por Badiou: la de ser filósofos y, a la vez, escritores.

Foucault y Rancière pertenecen al centro experimental de Vincennes (Paris VIII) creado inmediatamente después de mayo 68 y ambos comparten esa vocación polémica, a la vez filosófica y política que allí se manifestó, en vínculo con distintas corrientes marxistas principalmente no comunistas: althusserianos-maoístas (Badiou, Rancière), trotskistas (Bensaid), libertarios... sumándose a estas vanguardias Deleuze y Lyotard (Déotte, pp. 91 y 92).

Rancière describe bien las tendencias o novedades teóricas de esos tiempos y espacios. Dice: "lingüistas o antropólogos estructuralistas, filósofos althusserianos, psicoanalistas lacanianos, sociólogos formados en la escuela de Bourdieu y profesores de literatura instruidos por la semiología de Roland Barthes y la 'teoría literaria' del grupo 'Tel Quel'" (2013, p. 145). Particularmente importante fue la relectura del marxismo que hace Althusser (maestro éste del joven Rancière y amigo a su vez de Foucault) y cuyo posterior distanciamiento será decisivo para las nuevas exploraciones que efectúan ${ }^{3}$. En estos ambientes, Rancière reconoce su deuda con Foucault. En relación con el tipo de sujeto que se deja ver en los tiempos del dominio de la burguesía, aquel asistido, formado, vigilado, medido, y no el "sujeto triunfador del humanismo", Rancière menciona las enseñanzas de Foucault en el Collège de France (2013 p. 24).

No es casual, entonces, que los una cierta similitud o sintonía "metodológica", se podría decir. Entre, por ejemplo, El maestro ignorante (1987) de Rancière y la más alejada Arqueología del saber (1969) de Foucault, se aprecia un mismo interés por “describir"

2 No sin extrañeza, Vincent Descombes señala que "la postura política es y sigue siendo en Francia la prueba decisiva: es lo que debe revelar el sentido final de un pensamiento" (20). Sobre las relaciones entre Althusser y Foucault, así como sobre los aspectos filosóficos y políticos presentes antes y después de mayo 68, consultar Didier Eribon (1994). 
-más que "interpretar"- exterioridades, enunciados efectivos, correlaciones ${ }^{4}$. En ambos textos es visible la voluntad de escabullir la hermenéutica, los subtextos, las intenciones del autor, para destacar aquello que se juega en lo dicho y fenomenológicamente no más que en lo dicho. Ni en el examen de los enunciados foucaultianos, ni en la lectura que propone Jacotot del Telémaco (1699) de Fenelón, y que Rancière transcribe, habría un secreto por descubrir, un "sentido" que espera ser develado, un "detrás" de la página o del juego de los significantes, y que solo un inspirado filósofo o un convencido "maestro explicador" podría revelar. Dice Rancière refiriéndose al "método Jacotot”: “... es preciso descubrir que no hay nada oculto, no hay palabras bajo las palabras ni lengua que diga la verdad de la lengua" (2007, p. 40). Se une aquí la arqueología entendida como "reescritura", como reinscripción de lo dicho en Foucault, con una pedagogía cuya lectura/traducción del Telémaco transcurre por la superficie, por las distribuciones o conexiones no guiadas exteriormente y que el texto como tal manifiesta.

Esta aproximación fenomenológica o materialista al discurso toca, en efecto, a ambos autores. Se trata, dicho ahora desde otro ángulo, de un tipo de análisis que no escabulle la pesada inmaterialidad material o institucional del enunciado en Foucault (1988) o esa, cito, “interface creada entre 'soportes' diferentes, en los lazos tejidos entre el poema y su topografía o su ilustración” (2009, p. 16) en Rancière. Puestos en esta perspectiva es fácil constatar que lo que se evita es un tipo de análisis de carácter dualista, que diferencia el exterior del interior, la infra de la supra, la esencia de la apariencia (cfr. Jameson), para destacar "epistemes" en el momento "arqueológico" de Foucault o "poéticas del saber" en Ranciére, no tributarias ambas ni de las prerrogativas homogeinizadoras de la "lengua" (apelando a la acepción saussuriana) ni de un nivel que estuviese más allá del discurso o de las articulaciones de la gramática o de la retórica ${ }^{5}$. Este "materialismo" se expresa o está inscrito de entrada, aunque no siempre del mismo modo, en diversos tópicos: en la cuestión del cuerpo, de la anatomopolítica o en la relevancia que se le concede al nivel microfísico destacable principalmente en el momento "genealógico" de Foucault; y en la cuestión de la aisthesis, de la inmanencia, del nemeïn o del "reparto de lo sensible" en Rancière.

Dentro de este ámbito "metodológico", pero yendo más allá, cabe destacar igualmente el acceso que ambos autores hacen -y por tiempos largos- a las llamadas fuentes de "primera mano", a unos archivos polvorientos, a unos textos o testimonios olvidados que han dejado de leerse, aparentemente insignificantes. Textos que se

4 "Hay algo del esfuerzo arqueológico de Foucault que caló en mí", confiesa Rancière (2011a, p. 211).

5 Son muy conocidos los desplazamientos que se destacan y sobre todo se discuten en la obra de Foucault, más allá de la órbita lato sensu "estructuralista" de Las palabras y las cosas y La arqueología del saber que son textos de la segunda mitad de la década del 60. En el curso que dictó Gilles Deleuze sobre Foucault en la Universidad de Vincennes aborda críticamente los tres ejes clásicos del saber, el poder y la subjetivación. Cfr. Gilles Deleuze (2015, 2014 y 2015), Michel Foucault, "El sujeto y el poder” (1984), Miguel Morey (1991), “Introducción: La cuestión del método” y Mathieu Potte-Bonneville (2007, pp. 139 y sgs.). 
apartan del canon filosófico: desde los reglamentos de las prisiones o las muy precisas "normas" tendientes a orientar conductas en la sala de clases en Foucault, hasta los perdidos testimonios de obreros franceses del siglo XIX que rompen el "reparto" establecido entre la noche y el día en La noche de los proletarios en Rancière; desde el relato de una dramática vida incapaz de soportar la presión por definir su sexo en la hermafrodita Alexina B en Foucault, hasta esas aspiraciones de desidentificación, de ser otro, de vivir de otro modo, más allá del mito obrero o del lugar prefijado por el capital, publicados en La Ruche populaire en la primera mitad del XIX en Rancière ${ }^{6}$.

Sin temor a ensuciarse las manos con este tipo de fuentes, a relacionar registros distintos, o a desordenar las fronteras disciplinarias, Foucault y Rancière comparten la idea del intelectual indisciplinado, tan interesado por el presente como por la problematización de este interés, que dialoga con la literatura, la historia, la pintura, el cine y también con relatos de vida, archivos, etc. Un intelectual que no está dispuesto a renunciar a la crítica permanente de lo que somos, y que no se reconoce en una filosofía temerosa de redibujar sus espacios, de complejizar su centralidad o que se instala sin contratiempos ni tensiones, con cierta comodidad más bien, en el espacio de distribución o división de los saberes. Un cierto gusto por "épater les philosophes" se juega muy evidentemente aquí.

II. Demos ahora un giro al análisis. Si quisiéramos diferenciar los dos autores, se podría decir que mientras Foucault se interesó por la cuestión del "poder" bajo distintas acepciones, Rancière tendría a la "política" como uno de sus principales blancos, no asimilada ésta al ejercicio del poder. Una distinción que, si bien pedagógica, e incluso reforzada por Rancière, requiere de importantes matizaciones. Es lo que buscaré realizar en lo que viene.

Partamos recordando que en una entrevista Rancière afirmó que a Foucault no le interesaba la "subjetividad política", "al menos no a un nivel teórico. Foucault se ocupa del poder", concluyó7. Por otra parte, en sus "Diez tesis sobre la política" Rancière parece instalarnos bruscamente en otro escenario: "la política -dice- no es

6 Estos relatos no se pierden en su singularidad o dispersión. No se asumen como meras empiricidades. En Foucault, muestran determinadas operaciones del poder: lo que estas dejan ver o existir en los hombres infames, por ejemplo. O el cambio progresivo que comienza a darse tanto en la "inversión del eje de la individualidad" (el derecho a ser individuo, a contar con una historia personal) como en el "nuevo régimen de visibilidad" que se instala en la modernidad: el nuevo "derecho" a ser observado....y vigilado. Cfr. Vigilar y castigar. En Rancière estos relatos permiten a su vez apartarse de "modos usuales de comprensión del pensamiento obrero": de la tradición marxista para la cual la "consciencia obrera no podía desarrollarse sino gracias a la ayuda de una ciencia venida del exterior" como también de una suerte de contradiscurso que ha creído que esa consciencia "nacía de las tradiciones del oficio o de las formas de la cultura y de las sociabilidades populares" Cfr. Le philosophe et ses pauvres.

$7 \quad$ Cfr. Lazzarato 2010 / Rancière 2011a, pp. 123 y 124 / Tello, 14. 
el ejercicio del poder" (2006, tesis 1). La política es "algo específico" y que debe ser "definida por sí misma" (2006, tesis 1). Ella no se asimila ni a los "dispositivos" ni a las "relaciones de poder" de Foucault. Pero, ¿qué es lo propio de la política, entonces?, nos preguntamos. "La política-dice Rancière- es una ruptura específica de la lógica del arkhé" (2006, tesis 3), de cualquier mandato, título, comienzo o fundamento. Este es precisamente "el escándalo de la política", dirá Rancière (1996, p. 83): tanto el carácter infundado y no necesario de su acción como el tipo de ruptura que la constituye. La política deja en suspenso las validaciones del derecho, de la tradición, de la herencia, del contrato o pacto social original.

La piedra de toque es aquí la cuestión de la subjetividad. Y esto es particularmente claro en Ranciére. Para éste, la política requiere de unos operadores que-desdoblándose: siendo y no siendo lo que son bajo el dominio del capital- fracturan la comunidad del "arkhé", "mediante un nuevo modo de recuento de los no contados o de inclusión al excluido" (2011b, pp. 48 y otras). La política es indisociable de la irrupción de una subjetividad que se desclasifica, que rompe el lugar que le era asignado, y por esa vía desplaza, desordena o escinde la configuración, la unidad o el reparto del común sensible ${ }^{8}$. La política que es rara, ocasional, no necesaria, como los "enunciados" en Foucault, no se distingue entonces de la irrupción "de una parte de los que no tienen parte", de quienes haciendo valer el presupuesto de la "igualdad" de "cualquiera con cualquiera" reclaman su parte (Rancière 1996), verificando fácticamente dicha "igualdad".

Es importante hacer notar que la política tiene siempre como punto de arranque o de oposición lo que Rancière llama la "policía", aquel orden que se identifica con la estructuración o división de ese común sensible y simbólico que destacamos recién. La "policía" no se asimila aquí a un aparato o cuerpo estatal específico -significado relativamente reciente en Occidente- sino antes bien a su significado más antiguo, más cercano a la politeia, al gobierno o a la ordenación de la polis. Este era precisamente el uso que Foucault venía dando al término desde su Historia de la Locura y que tendrá un tratamiento más conceptual, junto al concepto de "gobierno", en Seguridad, territorio y población (1978).

Detengámonos brevemente en este último concepto, ya que este nos permite comenzar a salir de esa separación de aguas con que se inicia este segundo apartado, y que supone tanto una negación de la política en Foucault -"encerrada" en el "poder", reforzará Rancière (2011a, p. 124)- como la relación de esta con la subjetividad.

El concepto de "gobierno" entendido, en una de sus inflexiones, como gestión o dirección de las "conductas" y no como "autoridad pública", más dúctil o flexible

$8 \quad$ Laurent Jeanpierre y Dork Zabunyan advierten sobre la escisión que suele hacerse entre "un momento denominado 'político', seguido de un momento calificado de "estético", (Jacques Rancière. El método de la igualdad, 2014, p. 8) en cuanto ella pasa por alto el importante vínculo que se da en Rancière entre ambos momentos. Esto explica que en estas consideraciones tengamos presente, por citar dos textos importantes, tanto El Desacuerdo (1995) como El reparto de lo sensible (2000). 
que la noción de "dispositivo", y no reductible sin más a la noción de "soberanía", a la imposición de la ley y a las regularidades de la "disciplinas", como tampoco al modelo nietzscheano de la "guerra", me parece, en efecto, un primer acercamiento importante a lo que se podría entender como una "política" en Foucault (menos explícita que en Rancière) y que viene a complejizar unas "relaciones de poder" concebidas no tan mecánicamente.

En el seno de unas modalidades de acción que actúan sobre las posibilidades de acción y de deseo de los "otros" "“una acción sobre una acción" presente o futura, diría Foucault) se visibilizan unas tensiones e intersecciones abiertas a distintas formas, ámbitos y resistencias, que incluyen la participación del Estado y su racionalidad, una serie de prácticas de "gobierno" y también una diversidad de reacciones posibles. No es la determinación física ni la esclavitud sino la "libertad", la existencia de "sujetos libres" y activos, lo que sustenta o es la condición de unas "relaciones de poder" que son distintas, remarca Foucault, a los inmovilizados "estados de dominación" (2001 y 1994). En la estructuración de un campo posible "de acción sobre las acciones de los otros", no del todo ni necesariamente saturado, más bien inestable o modificable, tan vinculado al presente como a acontecimientos que pueden darse en el futuro, tienen lugar entonces, en el maremágnum de las tecnologías de "gobierno", y en el marco de un campo relacional, plural y competitivo de fuerzas, "todo un campo de respuestas", de prácticas de libertad y de "posibles invenciones", no solo las luchas más clásicas contra la formas de dominación y explotación sino también las que se dan contra las múltiples formas de sujeción (2001 y 2014). Para Foucault "todo es politizable, todo puede convertirse en política", pero no por la naturaleza de las cosas o porque el Estado directa o indirectamente puede manifestarse siempre, sino antes bien por la "indocibilidad reflexiva", intransigencia libertaria o, más claramente, por la "resistencia a la gubernamentalidad". La política es precisamente lo que nace -dirá Foucault en un manuscrito- de este específico enfrentamiento (Foucault 2014, p. 451. Ver también Foucault 2001, 2007b y Botticelli 2016).

En el marco principalmente de un régimen de "gobierno", las diversas formas que adquieren las relaciones entre técnicas o acciones de poder, resistencias o enfrentamientos, parecen tener un parecido de familia -aunque solo hasta cierto puntocon la contraposición o heterogeneidad entre la "policía" y la "política" rancièriana; todo lo cual abre el haz de la política (recordemos que para Foucault "todo puede convertirse en política"), no quedando ésta reducida ni a un dominio o racionalidad específica, siempre igual a sí mismo, a la weberiana, ni a una racionalidad global, unidimensional o ya establecida, a la frankfurtiana. Es el tipo de lectura o acercamiento entre Foucault y Rancière que he creído percibir en la compilación realizada por Andrés Tello (2016) y que el mismo refuerza en su artículo de 2017, y que me ha servido para la segunda

9 No habría que creer que un régimen de poder reemplaza sin más, como las epistemes, a otro régimen de poder. Aprendida ya la lección de Las palabras y las cosas, ahora para Foucault estos regímenes pueden combinarse formando un triángulo, por ejemplo, entre "soberanía”, “disciplina” y "gestión gubernamental” (2014, p. 135). 
parte de este ensayo como una importante fuente de inspiración. Este último ajuste entre Foucault y Rancière, que no es total, no se aplicaría al análisis de Mauricio Lazzarato de esta compilación, quien muy pertinentemente introduce como parte constituyente de la subjetivación política foucaultiana una "diferenciación ética", el "decir veraz" (la parrhesía), no exento de riesgos personales y políticos. Este "decir veraz" lleva o supone una fuerza o potencia de autoposicionamiento (la dynasteia) que es algo más que la presuposición de la "igualdad" como condición de la política en Rancière.

Del mismo modo, hay que señalar que en la concepción de la "política" en Rancière, en su contraposición o heterogeneidad con la "policía" como subrayamos, hay componentes que les son muy originales o propios: tal como la fractura del orden sensible -la "separación de lo sensible consigo mismo" dirá-, la impropiedad o anomalía del gesto político en cuanto "aloja un mundo en otro" (2006, tesis 8), el supuesto de la igualdad recién indicado, el modo como el nemeïn funda el nomos comunitario (2006, tesis 8), la revalorización de la democracia (Ruiz 2002, p. 79), así como la compleja problematización que realiza sobre el consenso y el disenso, entre otros componentes importantes de su renovación política y estética a la vez.

Si se considera pertinente la presente pesquisa, la de precisar una "política" en Foucault no impedida de extender su abanico (todo es politizable" señaló), no divorciada de "un campo de interacciones" (2007b, p. 34) ni de la cuestión de la "subjetividad", es revelador ese "desplazamiento teórico" que confiesa en su Historia de la sexualidad Tomo II (cuya primera edición en francés es de 1984), donde el desafío que representa la apropiación/construcción de sí ya no será desconocido sino antes bien potenciado por acciones prácticas, que caen en un amplio espectro de dominios (la dietética, la económica, la erótica), todos politizables en su diálogo con códigos y reglas de conducta, en el esfuerzo más global por avanzar hacia una "estética de la existencia" (1990, p. 15) que no habría que confundir con el solipsismo o el ensimismamiento sino como forma de apropiación e incluso de resistencia.

La construcción de un "actuar" sobre sí y los otros une su interés por la moral greco-romana con aquella "autonomía", "actitud" crítica y problematizadora del presente que percibe como característica del mundo ilustrado-moderno en Kant (Foucault, 2007b / Revel, 2002). En ese "actuar" y en esta "actitud" se halla uno de los componentes de lo que se podría reconocer más filosóficamente como una "política” en Foucault, no disociada del acceso a un régimen de veridicción no ajeno a transformaciones o reconversiones importantes en los sujetos mismos, por un lado, y a ese campo de tensiones e interacciones precipitantes que destacamos más arriba, por otro. En el paso que va del disciplinamiento o de las técnicas y acciones de gobierno al actuar soberano o independiente, y que asume además riesgos parrhesiásticos como recordaba Lazzarato, se localiza - me parece- uno de los gestos que identifican la activa, cambiante, diversa y tensa composición de fuerzas, irremediablemente política, que destaca Foucault.

Desmenucemos algo más. Según Etienne Tassin, hay dos momentos en la obra de Foucault que habría que entender como complementarios. En un primer momento, dice, "se trata de analizar las formas de sujeción impuestas por las relaciones de poder a los individuos que las padecen (41). En un segundo momento, continúa, "la 
subjetivación designa los procedimientos por los que un individuo se apropia de sí, se transforma él mismo en sujeto de sus propias prácticas" (41). Al sujeto sometido le sucede un "proceso de subjetivación", no un estado definitivo, más bien un devenir, muy abierto, no preestablecido, que para desprenderse de la sujeción tiene que habérselas necesariamente con unas objetivaciones. En la relación indisociable entre subjetivación y objetivación se expresa -en la propia relación o enfrentamiento habría que subrayarotro modo de destacar la dimensión política presente en Foucault.

Adicionalmente, habría que recordar que Foucault en una de sus últimas entrevistas ("El sujeto y el poder") hizo una relectura de sus investigaciones, desplazando la cuestión del poder en beneficio del tema del "sujeto" en su relación con diversos modos de “objetivación”, las “prácticas divisorias” entre otros (1984, p. 241). Una puntualización que, aun cuando podría entenderse como una más de las categorizaciones que él mismo propuso a unas investigaciones siempre en curso, hacía justicia a los seminarios que venía dictando en el Collège de France y que se abría a una hermenéutica e "inquietud de sí" que, extendiendo en este nuevo ámbito la noción de "gobierno" (Revel, p. 40), es de reveladoras consecuencias en los campos de la ética y de la política. Es precisamente en esta parte de su obra donde Foucault enfatiza las posibilidades que tiene el sujeto de devenir otro, de transformarse, en el difícil vencimiento de unas relaciones o imposiciones de distinto tipo que niegan estas posibilidades: un entramado que no permite divorciar a Foucault de su interés compartido por el sujeto, su "gobierno" y los factores que le están adheridos, evitando así que sea el "poder del poder" quien diga la "última palabra" (Deleuze 2015 / Castro, p. 177).

En el marco de unas dinámicas o interacciones que no desatiende entonces los procesos de constitución de los sujetos, los dos autores examinados se vuelven a encontrar en cierto sentido, tal como parece adelantar la compilación que he usado como referencia. Y esto porque el "proceso de subjetivación" indicado más arriba no es separable de una dinámica intensa, conflictiva, siempre relacional, de "desujeción", de desestabilización de determinados regímenes de verdad y de reconstrucción de sí en Foucault, así como de "desclasificación” y desorganización de ese reparto o común sensible que Rancière asimila a la "policía"10.

10 De la compilación de Andrés Tello (2016), ver especialmente el Preámbulo y los artículos de Samuel Chambers, Maria Muhle e Isabell Lorey publicados en esta compilación. Si el acento en el caso de Foucault está puesto aquí en una concepción de la "política" que tiende a subrayar principalmente las prácticas de "desujeción” y "subjetivación", en este artículo -sin dejar de reforzar, como se ve, estas importantes o decisivas prácticas- la mirada complementa o pone el acento también (es solo un acento no una alternativa distinta) en el campo estratégico y gubernamental de las "acciones" y "reacciones" mismas, actuales o eventuales, en el marco de "relaciones de poder" concebidas de un modo menos determinista (Foucault 2007b, pp. 33 y 34). Cabría preguntar si el caso de Rancière es distinto, quien distingue nítidamente "lo político" (el encuentro de dos procesos distintos: la "policía" y la "política") de la "política" más directamente vinculada-nunca en solitario, siempre en tensión con los órdenes policiales-con reconversiones subjetivas y estético-simbólicas. 
El o los sujetos no se conciben aquí como principios autónomos, previos a los poderes, a distintas objetivaciones y desplazamientos, al punto de que no hay procesos de resubjetivación o de conversión sin el consiguiente desprendimiento o emancipación de las sujeciones dadas: la búsqueda o el reclamo, por un lado, de otros modos de ser gobernados -no "por ellos" ni para "estos" fines- a contrapelo de la pastoral cristiana y de la posterior explosión, multiplicación, diversificación y laicización de las "artes de gobernar" en Foucault (2007, pp. 7 y otras) $)^{11}$, así como, por otro lado, la redistribución o desplazamiento de las posiciones (de los cuerpos, de las miradas, de los relatos o de los conceptos) en la creación de unas "escenas" estético-sensibles que se articulan no aisladamente sino refigurando o desasiéndose, no siempre de la misma manera o con el mismo alcance, de determinadas estructuraciones o regímenes de experiencia en Rancière (2018).

Así lo que inicialmente habíamos separado (el poder, por un lado, y la política, por el otro) se puede reformular bajo estas nuevas condiciones epistemológicas y políticas. Y si bien es posible detectar en distintos ámbitos complicidades importantes, tal como se ha buscado demostrar en este artículo, es claro, sin embargo, -y esto lo prueba la posibilidad misma de intercambio que hemos hecho- que en las trayectorias intelectuales examinadas, sin menoscabo de sus intertextualidades, no sería permitido arrebatarles su singularidad.

\section{Referencias bibliográficas}

Badiou, Alain (2014), La aventura de la filosofía francesa. A partir de 1960. Santiago de Chile: LOM Editores. (2010), La filosofía, otra vez. España: Errata Naturae. (1998), Petit manuel d'inesthétique. France: Seuil. (2007), Justicia, filosofía y literatura. Argentina: Homo Sapiens.

Botticelli, Sebastián (2016), "La gubernamentalidad del Estado en Foucault: un problema moderno", Praxis Filosófica 42. Colombia, enero-junio: pp. 83-106. Consultado el 7 mayo 2020. http://www.scielo.org.co/pdf/pafi/n42/n42a04.pdf

Castro Orellana, Rodrigo (2008), Foucault y el cuidado de la libertad. Ética para un rostro de arena. Santiago de Chile: LOM Editores.

Deleuze, Gilles (2015, 2014 y 2015), El Saber / El Poder / La Subjetivación. Buenos Aires: Cactus.

11 La voluntad de "desprenderse", de pensar, actuar o ser de otro modo es una constante en Foucault, ya visible en su momento "arqueológico". Voluntad que no habría que confundir con "procesos de liberación" que, aunque en ocasiones indispensables, pudiesen esconder, en el ámbito de la sexualidad por ejemplo, "fondos" o "naturalezas" enmascaradas o alienadas por mecanismos de "represión" así como nostalgias de reconciliación (1994, pp. 107 y 108). 
Déotte, Jean-Louis (2012), “¿Qué es un aparato estético?” Benjamin, Lyotard, Rancière. Santiago de Chile: Ediciones Metales Pesados.

Descombes, Vincent (1982), Lo mismo y lo otro. Cuarenta y cinco años de filosofía francesa (1933-1978). Madrid: Ediciones Cátedra.

Eribon, Didier (1994), Michel Foucault et ses contemporains. France: Fayard.

Foucault, Michel (2015), La gran extranjera. Para pensar la literatura. Argentina: Siglo Veintiuno Editores.

(1988), La arqueología del saber. México: Siglo Veintiuno Editores. (2007a), "Herculine Barbin llamada Alexina B.”. España: Talasa. (2001), "El sujeto y el poder" (1984), en Hubert L. Dreyfus y Paul Rabinow. Más allá del estructuralismo y la hermenéutica. Buenos Aires: Nueva Visión. Veintiuno.

(1990), Vigilar y castigar. Nacimiento de la prisión. México: Siglo (2014), Seguridad, territorio, población. México: Fondo de Cultura Económica.

(2009), La hermenéutica del sujeto. Argentina: Fondo de Cultura Económica. (1994), Hermenéutica del sujeto. Madrid: La Piqueta.

(1990), Historia de la sexualidad 2. El uso de los placeres. México: Siglo XXI.

(1992), Genealogía del racismo. Uruguay: Altamira. (2007b), Sobre la Ilustración. España: Tecnos.

Jameson, Fredric (1992), El posmodernismo o la lógica cultural del capitalismo avanzado. Argentina: Paidós.

Lazzarato, Maurizio (2010), "Une lectura parallèlle de la democratie: Foucault et Rancière". Internet. Consulta realizada el 4 de mayo 2020. http:// lesilencequiparle.unblog.fr/2010/04/14/enonciation-et-politique-une-lectureparallele-de-la-democratie-foucault-et-ranciere-maurizio-lazzarato/

Lorenzini, Daniele (2018), Entrevista: "Michel Foucault: prácticas de libertad y políticas del decir veraz", Dorsal. Revista de Estudios Foucaultianos 5: 141154.

Ossandón B., Carlos (2017), Gobierno y desacuerdo. Diálogos interrumpidos entre Foucault y Rancière. Andrés Tello, ed. (2016), Hybris. Revista de Filosofía. Vol. 8, $\mathrm{N}^{\circ}$ 2: 235-239.

Morey, Miguel (1991), "Introducción: La cuestión del método", en Michel Foucault, Tecnologías del yo y otros textos afines. Barcelona: Paidós.

Pereira Perdomo, Penélope, "Herencia de Foucault en Rancière: arqueología y política". Trabajo inédito presentado en el Seminario: "Foucault y Rancière" impartido por el prof. Carlos Ossandòn B. Universidad de Chile, Departamento de Filosofía, Primer semestre 2016. 
Potte-Bonneville, Mathieu (2007), Michel Foucault, la inquietud de la historia. Argentina: Manantial.

Rancière, Jacques (2013), La lección de Althusser. Santiago de Chile: LOM Editores. (2007), El maestro ignorante. Cinco lecciones sobre la emancipación intelectual. Argentina: Libros del Zorzal.

(1992), Les noms de l'histoire. Essai de poétique du savoir. France: Seuil. (2009), El reparto de lo sensible. Santiago de Chile: LOM Editores.

(2012), La nuit des prolétaires. Archives du rêve ouvrier. Espagne: Pluriel.

(2007), Le philosophe et ses pauvres. France: Flammarion.

(2014), El método de la igualdad. Conversaciones con Laurent Jeanpierre y Dork Zabunyan. Buenos Aires: Nueva visión.

(2011a), El tiempo de la igualdad. Diálogos sobre politica y estética.

España: Herder.

(2006), Política, policía, democracia. Santiago de Chile: LOM Editores. (1996), El desacuerdo. Política y filosofía. Buenos Aires: Nueva Visión. (2011b), Momentos políticos. España: Clave Intelectual. avec Adnen Jdey (2018), La méthode de la scène. Europe: Lignes.

Revel, Judith (2002), Le vocabulaire de Foucault. Paris: Ellipses.

Ruby, Christian (2010), Rancière y lo político. Argentina: Prometeo Libros.

Ruiz, Carlos (2002), "Sujeto y política en la filosofía política contemporánea”, en VVAA, Identidades y sujetos. Para una discusión latinoamericana. Santiago de Chile, Ediciones Facultad de Filosofía y Humanidades de la Universidad de Chile.

Tassin, Etienne (2012), "De la subjetivación política. Althusser / Rancière / Foucault / Arendt / Deleuze”, Revista de Estudios Sociales 43: 36-49. Bogotá

Tello, Andrés, ed. (2016), Gobierno y desacuerdo. Diálogos interrumpidos entre Foucault y Rancière. Viña del Mar: Comunes.

(2017), "La política de los modos de subjetivación. Consideraciones a partir de (des)encuentro entre Foucault y Rancière", Pensamiento al margen. Revista digital 7. Consultada 6 de mayo 2020.

Vega, Francisco y Valerio Rocco, ed. (2018), Estética del disenso. Políticas del arte en Jacques Rancière. Santiago de Chile: Doble Ciencia.

Yuing, Tuillang (2004), "Presentación a "La difícil herencia de M. Foucault", de Jacques Rancière", Paralaje 11: 201. 